\section{Fatores associados ao tracoma em área hipoendêmica da Região Sudeste, Brasil}

\author{
Factors associated with trachoma \\ in a low-endemic area in Southeast Brazil
}

\footnotetext{
1 Faculdade de Enfermagem Universidade de Santo Amaro, São Paulo, Brasil.

2 Faculdade de Saúde Pública, Universidade de São Paulo, São Paulo, Brasil.

3 Centro de Vigilância Epidemiológica, Secretaria de Estado da Saúde de São Paulo, São Paulo, Brasil.

Correspondência E. A. Waldman

Faculdade de Saúde Pública, Universidade de São Paulo. Av. Dr. Arnaldo 715, São Paulo, SP

01246-904, Brasil. eawaldma@usp.br
}

\section{Abstract}

A case-control study matched by age and school ( $n=121$ pairs) was carried out among pre-elementary and elementary students from low-income families living in Greater Metropolitan São Paulo, Brazil, with the objective of investigating factors associated with trachoma in a low prevalence area. The case definition for trachoma was that proposed by the World Health Organization. The dependent variable was trachoma and the independent variables were social stratum, housing of migrants from endemic areas, facial hygiene habits, head of family's schooling, access to potable water, contact with another case in the family, and slum residence. Unadjusted and adjusted odds ratios (OR), with their respective 95\% confidence intervals (95\%CI), were estimated by conditional logistic regres sion. In the multivariate analysis, belonging to the poorest social stratum $(O R=8.21 ; 95 \% C I$ : $1.50-44.81)$, housing people from endemic areas $(O R=2.44 ; 95 \% C I: 1.10-5.46)$, contact with another case in the family (OR $=2.52$; 95\%CI: 0.98 6.48), and facial hygiene habits (OR $=0.50$; 95\%CI: 0.26-0.98) were independently associated with trachoma.

Case-control Studies; Child Welfare; Trachoma
Rosa Kazuye Koda D'Amaral 1 Maria Regina Alves Cardoso 2 Norma Helen Medina ${ }^{3}$ Isabel Cristina Kowal Olm Cunha 1 Eliseu Alves Waldman ${ }^{2}$

\section{Introdução}

O tracoma é a segunda causa de cegueira em todo o mundo, mantendo-se em níveis hiperendêmicos em várias regiões da Ásia, África e Oriente Médio 1. Nas Américas existem focos localizados no sul do México, Guatemala, Bolívia, Peru e no Nordeste do Brasil 2.

A queda acentuada da incidência do tracoma em países industrializados pela melhoria das condições de vida e, especialmente, as de higiene, tem diminuído sua visibilidade como problema em saúde pública 3 .

Nas regiões mais desenvolvidas do Brasil assiste-se a fenômeno semelhante. No Estado de São Paulo, por exemplo, houve declínio acentuado da prevalência do tracoma desde meados da década de 1940, permitindo que alguns técnicos considerassem-no "erradicado" 4. No entanto, sua presença volta a ser assinalada nos anos 80 , mas com baixa endemicidade. Luna et al. 5 verificaram na região noroeste do Estado de São Paulo prevalência de 2,1\% da forma inflamatória do tracoma, atingindo principalmente as populações rurais e periurbanas.

Poucos estudos têm detalhado o comportamento do tracoma em áreas hipoendêmicas. As publicações focalizando a região metropolitana de São Paulo sugerem uma baixa prevalência, porém os estudos não foram conclusivos quanto aos principais fatores de risco, tornan- 
do oportuno o desenvolvimento de pesquisa com essa finalidade $6,7,8$.

O objetivo desta investigação foi identificar os fatores associados ao tracoma entre escolares e pré-escolares residentes em comunidades de baixa renda do Município de São Paulo, com a finalidade de oferecer subsídios ao aprimoramento de estratégias de controle do tracoma em áreas hipoendêmicas.

\section{Casuística e métodos}

\section{Delineamento}

O delineamento utilizado foi o de estudo casocontrole, no qual os casos foram comparados com igual número de controles pareados por idade e por escola.

\section{Área e população de estudo}

O estudo foi desenvolvido em nove distritos situados em região da área metropolitana de São Paulo, com população estimada para o ano do estudo em 1.214.044 habitantes. Esses distritos foram selecionados por abranger expressiva parcela da população da área metropolitana, pela alta proporção de domicílios inadequados e de famílias de baixa renda e pelo elevado número de migrantes, caracterizando-os como área vulnerável ao tracoma. Os Índices de Desenvolvimento Humano (IDH) desses distritos variavam de 0,245 a 0,759 , sendo que em seis distritos esse indicador estava abaixo de 0,50 9 .

A população de estudo foi constituída pelos alunos matriculados em seis pré-escolas e oito escolas de ensino fundamental e médio, todas elas públicas. Tais unidades de ensino fizeram parte de uma amostra representativa das escolas públicas do Município de São Paulo, selecionada para um inquérito que teve como objetivo estudar a prevalência de tracoma entre escolares no período de maio a agosto de 1999.

As 14 escolas públicas incluídas neste estudo possuíam 8.727 escolares matriculados e que as freqüentavam regularmente, sendo que o inquérito abrangeu a totalidade desses escolares. Nas pré-escolas a idade das crianças variava de 5 a 7 anos, enquanto que nas escolas de ensino fundamental e médio, de 7 a 18 anos.

\section{Definição de casos e controles}

Definiu-se como caso os estudantes matriculados em uma das 14 escolas selecionadas e residentes em um dos nove distritos do Município de São Paulo incluídos no estudo (Campo Gran- de, Santo Amaro, Cidade Ademar, Pedreira, Cidade Dutra, Socorro, Grajaú, Parelheiros e Marsilac) que ao exame clínico, realizado durante o inquérito efetuado no período de maio a agosto de 1999, tivessem apresentado sinais de tracoma ativo de acordo com critérios propostos pela Organização Mundial da Saúde (OMS) 10. Segundo tais critérios, as formas de tracoma ativo foram classificadas em cinco graus de intensidade:

- Inflamação tracomatosa folicular (TF) - na conjuntiva tarsal superior com um mínimo de cinco folículos de $0,5 \mathrm{~mm}$ de diâmetro;

- Inflamação tracomatosa difusa intensa (TI) - espessamento inflamatório pronunciado da conjuntiva que encobre mais da metade dos vasos tarsais profundos;

- Cicatrização conjuntival tracomatosa (TS) - a presença de cicatrizes na conjuntiva tarsal que são facilmente visíveis como linhas, bandas ou fios (fibroses) na conjuntiva tarsal;

- Triquíase tracomatosa (TT) - cílios invertidos (triquíase), quando pelo menos um dos cílios atrita o globo ocular;

- Opacificação corneana (CO) - quando esta é facilmente visível sobre a pupila.

A verificação dos sinais de tracoma ativo foi feita clinicamente, pelo exame da pálpebra superior evertida e da conjuntiva tarsal com lupa binocular de 2,5 vezes de aumento e iluminação adequada (artificial com lanterna de bolso ou iluminação natural), de acordo com a técnica de Dawson et al. 11 .

Os exames foram feitos por quatro profissionais, treinados e padronizados para a identificação de sinais de tracoma ativo, segundo critério proposto pela OMS 10. Tais profissionais apresentavam à época da pesquisa, ao menos três anos de experiência como membros da equipe da Secretaria de Estado da Saúde de São Paulo responsável por inquéritos de tracoma, e haviam sido habilitados para tal atividade após treinamento específico no qual apresentaram 80,0\% ou mais de concordância em relação ao especialista, tomado como padrão de referência. Todas as crianças com diagnóstico de tracoma inflamatório foram tratadas segundo a terapêutica indicada.

Para cada caso foi selecionado um controle entre escolares que se mostraram negativos aos exames para identificação de casos de tracoma efetuados à época do inquérito e no momento da seleção, de acordo com a definição de tracoma estabelecida pela pesquisa. Os controles foram pareados por ano de nascimento e por freqüência à mesma escola e classe dos casos, sendo selecionados por meio de amostragem probabilística. Todos os controles deve- 
riam ser residentes num dos nove distritos incluídos no estudo.

Considerou-se perda para casos e controles sempre que: (i) os pais não fossem encontrados em três visitas sucessivas ao domicílio; (ii) quando os pais ou responsáveis se apresentassem impossibilitados de responder ao questionário; (iii) ou ainda quando estes se recusassem a participar da pesquisa.

O tamanho da amostra foi estimado em 150 casos e igual número de controles, para que fosse possível atingir nível de 5,0\% de significância, poder de $80,0 \%$ para detectar uma odds ratio igual ou maior que 2 para prevalência de exposição nos controles variando de $30,0 \%$ a $70,0 \%$.

Para a classificação socioeconômica utilizaram-se os critérios propostos por Almeida \& Wilckerhausers 12 , que se fundamentam na escolaridade do chefe da família e na posse ou acesso a alguns bens móveis e serviços, estabelecendo-se uma escala que permite a classificação das famílias em cinco estratos sócio-econômicos: A, B, C, D e E, sendo que o primeiro é o que apresentava melhores indicadores.

Foi realizada visita domiciliar a todos os casos selecionados e aos respectivos controles, com aplicação de questionário estruturado e realização do exame ocular dos comunicantes menores de dez anos. Todos os dados obtidos foram digitados em microcomputador utilizando-se o programa Epi Info, versão 6.4, sendo que a análise estatística foi efetuada com o programa Stata, versão 7 .

A variável dependente foi a presença do tracoma e as independentes: características de habitação; forma de acesso à água no domicílio; estrato sócio-econômico a que pertence a família; existência de outro caso na família; hospedar com freqüência pessoas de regiões endêmicas de tracoma; escolaridade do chefe da família e higiene facial, esta última analisada segundo o número de vezes que a criança lavava o rosto por dia.

A existência de associação entre tracoma e as exposições de interesse foi investigada pelas estimativas não ajustadas e ajustadas da $o d d s$ ratio, com os respectivos intervalos de $95 \%$ de confiança (IC95\%), usando regressão logística condicional para considerar o efeito do pareamento. A significância estatística das variáveis nos modelos foi avaliada pelo teste de razão de verossimilhança 13 .

Esta pesquisa foi aprovada pela Comissão de Ética da Faculdade de Saúde Pública da Universidade de São Paulo. As crianças somente foram examinadas após o consentimento dos pais ou responsáveis, o mesmo procedimento foi observado em relação à aplicação dos questionários.

\section{Resultados}

Foram identificados 150 casos de tracoma da forma folicular, no período de maio a agosto de 1999 nas escolas selecionadas; 14 (9,3\%) foram excluídos por não residirem na área de estudo. Em 15 (11,0\%) dos 136 casos restantes não foi possível realizar a entrevista com os pais ou responsáveis pelos seguintes motivos: sete por mudança de endereço, quatro por endereço não localizado, dois por não residirem no endereço indicado e outros dois por ausência de adulto no momento da visita em três tentativas sucessivas. Dos 204 controles inicialmente selecionados, 83 não foram entrevistados pelos mesmos motivos descritos acima para os casos. Assim, a amostra final foi constituída de 121 pares de casos e controles.

A idade dos escolares que participaram deste estudo variou entre 4 e 18 anos, com mediana de dez anos. Houve um discreto predomínio do sexo masculino.

A Tabela 1 apresenta a distribuição de casos e controles segundo características sócio-demográficas.

Tanto casos como controles pertenciam a famílias que figuravam em estratos sócio-econômicos de menor renda (cerca de 90,0\% pertenciam às classes $\mathrm{C}, \mathrm{D}$ e E). Aproximadamente $77,0 \%$ dos pais dos escolares eram migrantes originários das regiões menos desenvolvidas do país, sendo que $56,0 \%$ e $52,0 \%$ dos pais, respectivamente dos casos e dos controles, nasceram na Região Nordeste, área endêmica de tracoma. No entanto, cerca de $67,0 \%$ das famílias de casos e controles residiam no Estado de São Paulo há 15 anos ou mais.

Quanto à educação, 4,0\% e 3,0\% dos chefes de família, respectivamente de casos e controles, não possuíam nenhuma instrução. Com referência às condições habitacionais de casos e controles, 98,0\% residiam em domicílios de alvenaria; a mediana do número de pessoas por domicílio foi de 3,8 pessoas/domicílio; praticamente 100,0\% dos domicílios possuíam acesso à água de abastecimento público e coleta de lixo de três vezes por semana; e em torno de 79,0\% tinham acesso a serviço de esgotamento sanitário. Como indicador de acesso à informação, vale salientar que somente $7,0 \%$ dos casos e $3,0 \%$ dos controles não possuíam televisão no domicílio.

$\mathrm{Na}$ análise bivariada pareada, as variáveis que se mostraram associadas ao tracoma com valor de $\mathrm{p}<0,05$ (Tabela 2) foram: pertencer ao grupo sócio-econômico de menor poder aquisitivo (estrato E); hospedar pessoas da região nordeste; higiene do rosto com freqüência igual ou maior a três vezes ao dia; e existência de outro 
Distribuição de casos e controles de tracoma segundo variáveis sócio-demográficas. São Paulo, Brasil, 1999/2000.

\begin{tabular}{|c|c|c|c|c|}
\hline \multirow[t]{2}{*}{ Variáveis } & \multicolumn{2}{|c|}{ Casos } & \multicolumn{2}{|c|}{ Controles } \\
\hline & $\mathrm{n}$ & $\%$ & $\mathrm{n}$ & $\%$ \\
\hline \multicolumn{5}{|l|}{ Faixa etária (em anos) } \\
\hline $4-6$ & 25 & 20,7 & 25 & 20,7 \\
\hline $7-14$ & 90 & 74,4 & 90 & 74,4 \\
\hline $15-18$ & 6 & 4,9 & 6 & 4,9 \\
\hline \multicolumn{5}{|l|}{ Sexo } \\
\hline Masculino & 62 & 51,2 & 61 & 50,4 \\
\hline Feminino & 59 & 48,8 & 60 & 49,6 \\
\hline \multicolumn{5}{|l|}{ Município de nascimento } \\
\hline São Paulo & 102 & 84,3 & 105 & 86,8 \\
\hline Outras cidades & 19 & 15,7 & 16 & 13,2 \\
\hline \multicolumn{5}{|l|}{ Estado de nascimento } \\
\hline São Paulo & 107 & 88,4 & 111 & 91,7 \\
\hline Outros Estados & 14 & 11,6 & 10 & 8,3 \\
\hline \multicolumn{5}{|l|}{ Tempo de residência em São Paulo } \\
\hline Sempre morou & 97 & 80,2 & 93 & 76,9 \\
\hline Menos de 4 anos & 4 & 3,3 & 11 & 9,1 \\
\hline 5 anos e mais & 20 & 16,5 & 17 & 14,0 \\
\hline \multicolumn{5}{|l|}{ Domicílio em favela } \\
\hline Não & 78 & 65,5 & 87 & 71,9 \\
\hline Sim & 43 & 35,5 & 34 & 28,1 \\
\hline \multicolumn{5}{|l|}{ Tipo de habitação } \\
\hline Casa de alvenaria & 68 & 56,2 & 70 & 57,8 \\
\hline Casa de alvenaria em favela & 43 & 35,5 & 34 & 28,1 \\
\hline Conjuntos residenciais populares & 8 & 6,6 & 15 & 12,4 \\
\hline Outros & 2 & 1,7 & 2 & 1,7 \\
\hline \multicolumn{5}{|l|}{ Água no domicílio } \\
\hline Contínua & 100 & 82,6 & 109 & 90,1 \\
\hline Intermitente & 21 & 17,4 & 12 & 9,9 \\
\hline \multicolumn{5}{|l|}{ Condição de ocupação da habitação } \\
\hline Própria & 99 & 81,8 & 95 & 78,5 \\
\hline Alugada & 17 & 14,1 & 15 & 12,4 \\
\hline Outras & 5 & 4,1 & 11 & 9,1 \\
\hline \multicolumn{5}{|l|}{ Número de pessoas no domicílio } \\
\hline $2-4$ & 61 & 50,4 & 51 & 42,2 \\
\hline $5-7$ & 51 & 42,2 & 61 & 50,4 \\
\hline $8 \mathrm{e}+$ & 9 & 7,4 & 9 & 7,4 \\
\hline \multicolumn{5}{|l|}{ Chefe da família } \\
\hline Pai & 76 & 63,3 & 84 & 69,4 \\
\hline Mãe & 30 & 25,0 & 22 & 18,2 \\
\hline Outra pessoa & 14 & 11,7 & 15 & 12,4 \\
\hline \multicolumn{5}{|l|}{ Escolaridade do chefe da família } \\
\hline 1ㅇ grau completo ou mais & 37 & 30,0 & 47 & 38,8 \\
\hline 1o grau incompleto ou menos & 83 & 70,0 & 74 & 61,2 \\
\hline
\end{tabular}


Resultados do modelo final de regressão logística condicional com respectivas odds ratios (OR) não ajustadas e ajustadas, intervalos de confiança de 95\% (IC95\%) e valores de p. São Paulo, Brasil, 1999/2000.

\begin{tabular}{|c|c|c|c|c|c|}
\hline Variável & Casos & Controles & $\begin{array}{l}\text { OR não ajustada } \\
\text { (IC95\%) }\end{array}$ & $\begin{array}{l}\text { OR ajustada } \\
\text { (IC95\%) }\end{array}$ & $p^{*}$ \\
\hline Estrato sócio-econômico & & & & & 0,02 \\
\hline B & 13 & 15 & 1,00 & 1,00 & \\
\hline C & 56 & 63 & $1,06(0,44-2,56)$ & $1,30(0,40-4,45)$ & \\
\hline D & 32 & 37 & $1,12(0,44-2,85)$ & $1,94(0,54-7,03)$ & \\
\hline E & 20 & 6 & $4,11(1,16-14,54)$ & $8,21(1,50-44,81)$ & \\
\hline Hospedar pessoas de área endêmica & & & & & $<0,0001$ \\
\hline Não & 72 & 87 & 1,00 & 1,00 & \\
\hline Sim (Região Nordeste) & 41 & 23 & $2,40(1,17-4,80)$ & $2,44(1,1-5,46)$ & \\
\hline $\begin{array}{l}\text { Higiene do rosto (número } \\
\text { de vezes por dia) }\end{array}$ & & & & & 0,002 \\
\hline $1-2$ & 82 & 62 & 1,00 & 1,00 & \\
\hline $3 \mathrm{ou}+$ & 35 & 57 & $0,50(0,30-0,84)$ & $0,50(0,26-0,98)$ & \\
\hline $\begin{array}{l}\text { Existência de outro caso na família } \\
\text { (nos últimos } 6 \text { meses) }\end{array}$ & & & & & 0,04 \\
\hline Não & 96 & 110 & 1,00 & 1,00 & \\
\hline Sim & 25 & 11 & $2,55(1,20-5,50)$ & $2,52(0,98-6,48)$ & \\
\hline
\end{tabular}

* Teste da razão de verossimilhança.

caso na família nos últimos seis meses. Foi observada associação com significância estatística limítrofe entre tracoma e abastecimento de água intermitente no domicílio, $\operatorname{com} \mathrm{OR}=2,12$ (IC95\%: 0,92-4,92; $p=0,07$ ). Estiveram ainda associadas ao tracoma as variáveis: domicílio localizado em favela, com OR $=1,40$ (IC95\%: $0,81-2,40 ; p=0,22$ ) e escolaridade do chefe da família igual ao primeiro grau incompleto ou menos, com OR = 1,43 (IC95\%: 0,82-2,50; $\mathrm{p}=$ $0,21)$, porém sem significância estatística.

Na Tabela 2 são apresentadas as odds ratios ajustadas obtidas pelo modelo final de regressão logística condicional múltipla. As associações observadas nas análises bivariadas foram mantidas, com exceção do abastecimento de água no domicílio, do domicílio localizado em favela e da escolaridade do chefe da família, que perderam a significância estatística.

\section{Discussão}

Após um período de aparente desaparecimento, a partir dos anos 80, quando foram confirmados casos de tracoma inflamatório na região noroeste do Estado de São Paulo 5, estudos sucessivos têm mostrado a persistência de focos residuais no estado, tornando indispensável o desenvolvimento de um sistema de vigilância com o objetivo de identificar tendências, grupos e fatores de risco para o tracoma em área de baixa prevalência 14,15 .

$\mathrm{O}$ inquérito conduzido entre pré-escolares e escolares residentes em nove distritos da área metropolitana de São Paulo, que serviu de instrumento para a seleção de casos e controles para a presente investigação, mostrou uma prevalência de $1,7 \%$ de tracoma, variando de $0,5 \%$ a $3,9 \%$, conforme a unidade de ensino. A prevalência encontrada no mesmo inquérito, mas abrangendo uma amostra de todo o Município de São Paulo foi semelhante, situando-se em $2,2 \% 16$. Todos os casos identificados apresentavam a forma folicular.

A ausência de formas graves da doença e a baixa prevalência, verificadas nesse inquérito e em outras pesquisas realizadas na área metropolitana de São Paulo, a partir dos anos 90 7,8,17, nos permite caracterizar essa área como hipoendêmica e de baixo risco para formas cicatriciais e de opacificação da córnea associadas ao tracoma em face da pequena probabilidade de infecções repetidas 18 .

A baixa circulação da Chlamydia trachomatis encontrada na população em que seleciona- 
mos os casos e controles para nossa investigação é consistente com as condições de vida das famílias desses escolares, que se mostrou muito homogênea em relação a vários indicadores de saneamento e tipo de moradia, com infraestrutura urbana melhor do que aquela geralmente encontrada em áreas endêmicas do tracoma, onde diversas pesquisas apontam prevalências variando de $22,2 \%$ a $51,7 \%$, com a presença de casos de cegueira 19,20,21,22.

No presente trabalho, um fator fortemente associado ao tracoma, de acordo com a análise bivariada pareada, foi pertencer ao estrato sócio-econômico de menor poder aquisitivo, resultado consistente com inúmeros trabalhos desenvolvidos em diferentes países e condições ambientais, sugerindo a importância deste fator tanto em áreas hiper como hipoendêmicas 5,18,23.

Hospedar pessoas de região endêmica foi outra exposição que se mostrou associada ao tracoma, sugerindo que migrantes, mesmo quando há muito tempo integrados à vida da região metropolitana de São Paulo, mantêm vínculos sociais com sua comunidade de origem, e que este fator pode favorecer a reintrodução da C. trachomatis, mesmo em área que há décadas deixou de ser endêmica desse agente.

A elevada proporção dos pais dos escolares incluídos na pesquisa que nasceram em área endêmica do tracoma não constitui, por si, um risco, uma vez que os mesmos, em sua maioria, residiam no Estado de São Paulo há 15 anos ou mais. Tal afirmativa é fundamentada no fato de indivíduos originários de áreas endêmicas, ao migrarem para países livres do tracoma não apresentarem casos de recrudescência da doença ou sinais de persistência da infecção, sugerindo que esse fator não estaria associado, nesses indivíduos, a novos episódios de infecção pela C. trachomatis 24 .

A existência de outro caso na família nos últimos seis meses também apresentou associação com o tracoma. Tal resultado apresenta consistência com o fato da transmissão da C. trachomatis ocorrer com alguma freqüência mediante contato com roupas de cama e banho, ou ainda pelo próprio contato entre os membros de uma mesma família. Possivelmente a associação não se mostrou mais forte em virtude dessas famílias serem, em boa parte, pouco numerosas, com a mediana de 3,8 pessoas por domicílio, além de possuírem acesso quase universal à água, facilitando a higiene pessoal 25,26,27.

Duas exposições que mostraram fraca associação com o tracoma e ausência de significância estatística foram: escolaridade do chefe da família e piores condições habitacionais, to- mando o fato de "residir em domicílio localizado favela" como um indicador.

Nesses dois casos a ausência de significância estatística talvez possa ser explicada pelo tamanho da amostra, uma vez que, escolaridade do chefe da família e condições habitacionais têm sido apontadas como fatores associados ao tracoma em vários estudos $5,18,23$, fortalecendo a hipótese de que essas exposições sejam preditoras da doença. Vale lembrar que a melhoria das condições de vida tem acompanhado a tendência declinante do tracoma, em diferentes populações, mesmo na ausência de intervenções destinadas ao seu controle 28 .

Um fator protetor que se mostrou fortemente associado ao tracoma foi a higiene facial, resultado consistente com vários trabalhos 27,29. De certa forma, esse dado é coerente com a associação observada entre acesso à água intermitente e tracoma $(\mathrm{OR}=2,12 ; \mathrm{p}=0,07)$, que apesar da significância estatística limítrofe na análise bivariada, sugerindo influência do tamanho da amostra, tem sua plausibilidade biológica bem fundamentada na literatura 5,18,30.

Em geral condições inadequadas de higiene favorecem a transmissão da C. trachomatis por meio do contato com secreções 31. Estudo longitudinal realizado em dois momentos, num intervalo de seis anos, verificou que crianças que apresentaram higiene inadequada da face na primeira observação e adequada no acompanhamento, mostraram menor probabilidade de apresentar tracoma intenso do que aquelas com higiene inadequada nos dois momentos do estudo $(\mathrm{OR}=0,21) 32$.

Entre as possíveis limitações deste estudo, podemos apontar o menor valor preditivo positivo, em áreas de baixa prevalência da doença, do critério clínico de identificação de casos de tracoma ativo 10. Dessa forma, é possível que tenham ocorrido erros de classificação com a inclusão de casos falsamente positivos 26,33. Todavia se isso ocorreu, o efeito deste viés foi o de subestimar a odds ratio. Outra limitação pode ter origem na inclusão no estudo de casos prevalentes e não incidentes, o que induziria o viés de incidência-prevalência, que leva à diminuição, entre os casos, da proporção de expostos a fatores associados à duração do desfecho, diluindo a força da associação 34 . No entanto, acreditamos que em nosso estudo esse viés não deva ter influenciado de forma expressiva os resultados, pois casos e controles foram pareados para o principal fator que condiciona a duração do tracoma, a idade. Por outro lado, estudo recente estimou em 5,3 semanas a média do tempo de duração do tracoma na faixa etária incluída no presente estudo 35. Por fim, 
devemos também assinalar que em função das perdas, o tamanho da amostra tornou-se pequeno, determinando menor poder do estudo para encontrar, como significante, associações de menor intensidade e envolvendo exposições menos freqüentes.

Os resultados apresentados mostraram que em comunidades com baixa circulação da $C$. trachomatis podem persistir resíduos de casos e fontes de infecção nos segmentos mais pobres da população, que apresentam hábitos de higiene inadequados, e entre familiares de migrantes de áreas endêmicas que mantenham vínculo social com as suas comunidades de origem.

\section{Resumo}

Foi desenvolvido um estudo caso-controle pareado por idade e escola ( $n=121$ pares) entre escolares e pré-escolares residentes em área periférica da região metropolitana de São Paulo, Brasil, com o objetivo de investigar fatores associados ao tracoma em área de baixa prevalência. Foi utilizada a definição de caso proposta pela Organização Mundial da Saúde. Os casos foram identificados em inquérito prévio e os controles selecionados na mesma população, mediante sorteio entre os que não apresentavam a condição de interesse. A variável dependente foi tracoma e as independentes: estrato sócio-econômico, hospedar pessoas originárias de áreas endêmicas, hábitos de higiene, escolaridade do chefe da família, acesso à água, contato com outro caso na família e residir em favela. As odds ratios (OR) não ajustada e ajustada e os respectivos intervalos de de confiança de 95\%(IC95\%) foram estimados por regressão logística condicional. Mostraram-se independentemente associados ao tracoma: pertencer ao estrato social de menor poder aquisitivo $(\mathrm{OR}=8,21$; IC95\%: 1,50-44,81), hospedar pessoas originárias de área endêmica $(O R=2,44$; IC95\%: $1,1-5,46)$, contato com outro caso na família (OR = 2,52; IC95\%: 0,986,48) e higiene facial (OR =0,5; IC95\%: 0,26-0,98).

Estudos de Casos e Controles; Bem-estar da Criança; Tracoma
Considerando o elevado custo de inquéritos periódicos e os resultados apresentados, podemos recomendar para áreas de baixa endemicidade, semelhantes à estudada, a inclusão de treinamento de professores de pré-escola e do ensino básico na estratégia de controle do tracoma, com a finalidade de desenvolver atividades educativas visando à incorporação, pelas crianças, de hábitos rotineiros de higiene facial, focalizando principalmente menores de dez anos. Por outro lado, é importante estabelecer como rotina da rede básica de serviços, o tratamento com antibiótico de todos os casos com evidência clínica de tracoma ativo, assim como de seus familiares e contatos domiciliares.

\section{Colaboradores}

R. K. K. D'Amaral coordenou a pesquisa e participou de todas as suas etapas: elaboração do projeto, trabalho de campo, análise de dados e redação do artigo. M. R. A. Cardoso participou na elaboração do desenho da amostra, na análise de dados e redação do artigo. N. H. Medina coordenou o estudo de corte transversal, de cuja amostra foram selecionados os casos e controles do estudo caso-controle objeto deste artigo, e participou da revisão do texto final do artigo. I. C. K. O. Cunha participou na análise de consistência dos dados e auxiliou na revisão do artigo. E. A. Waldman participou na elaboração do projeto, análise e redação do artigo.

\section{Agradecimentos}

Maria Schunck de Morais, Yvone Marcondes Ribeiro de Andrade e Zilmar Moreira Xavier Ayabe, pela importante participação na coleta de dados realizando as visitas domiciliares.

\section{Referências}

1. Thylefors B, Négrel AD, Pararajasegaram R, Dadzie KY. Global data on blindness. Bull World Health Organ 1995; 73:115-21.

2. Programme for the Prevention of Blindness and Deafness, World Health Organization. Future approaches to trachoma control: report of a global scientific meeting. Geneva: World Health Organization; 1997.

3. Cook JA. Trachoma. Bull World Health Organ 1998; 76 Suppl 2:139-40. 
4. Freitas CA. Prevalência do tracoma no Brasil. Rev Bras Malar Doenças Trop 1976; 28:1-4.

5. Luna EJA, Medina NH, Oliveira MB, Barros OM, Vranjac A, Melles HHB, et al. Epidemiology of trachoma in Bebedouro, State of São Paulo, Brazil: prevalence and risk factors. Int J Epidemiol 1992; 21:169-77.

6. Campos CEG. Prevalência de tracoma entre préescolares e escolares moradores em favelas do bairro da Freguesia do Ó, Região Norte do Município de São Paulo, Estado de São Paulo, 1992 [Dissertação de Mestrado]. São Paulo: Escola Paulista de Medicina, Universidade Federal de São Paulo; 1992.

7. Caligaris LSA. Ocorrência de tracoma em população pré-escolar no subdistrito de paz da Bela Vista, município de São Paulo, 1995 [Dissertação de Mestrado]. São Paulo: Faculdade de Saúde Pública, Universidade de São Paulo; 1998.

8. Medina NH, Massaini MG, Azevedo CLB, Harima C, Prado M, Maluf S, et al. Vigilância epidemiológica do tracoma em instituição de ensino na Cidade de São Paulo, SP. Rev Saúde Pública 1998; 32:59-63.

9. Secretaria do Desenvolvimento, Trabalho e Solidariedade do Município de São Paulo. Desigualdade no Município de São Paulo: o IDH. São Paulo: Secretaria do Desenvolvimento, Trabalho e Solidariedade do Município de São Paulo; 2002.

10. Thylefors B, Dawson CR, Jones BR, West SK, Taylor HR. A simple system for assessment of trachoma and its complications. Bull World Health Organ 1987; 65:477-83.

11. Dawson CR, Jones BR, Tarizzo ML. Guia práctica de lucha contra el trachoma. Geneva: Organización Mundial de la Salud; 1981.

12. Almeida PL, Wickerhauser H. Finding a better socio-economic status classification system for Brazil. Marketing and Research Today 1991; 1: 240-50.

13. Clayton D, Hills M. Statistical models in epidemiology. Oxford: Oxford Science Publications; 1996.

14. Medina NH, Gentil RM, Oliveira MB, Sartori MF, Cabral JH, Vasconcelos MS, et al. Investigação epidemiológica do tracoma em pré-escolares e escolares nos municípios de Franco da Rocha e Francisco Morato - São Paulo. Arq Bras Oftalmol 1989; 57:154-8.

15. Medina NH, Oliveira MB, Tobin S, Kiil Jr. G, Mendonça MME, Barros OM, et al. The prevalence of trachoma in prescholl and school children in Olímpia, Guaraci and Cajobi, São Paulo, Brazil. Trop Med Parasitol 1992; 43:121-3.

16. Koizumi IK, D'Amaral RKK, Favaro EM, Morimoto WMM, Medina NH. Prevalência do tracoma no Município de São Paulo. Arq Bras Oftal 2000; 63 (4 Suppl):63.

17. Medina NH, Massaini MG, Azevedo CLB, Harima C, Prado M, Maluf S, et al. Vigilância epidemiológica do tracoma em instituição de ensino na Cidade de São Paulo, SP. Rev Saúde Pública 1998; 32:59-63.
18. Katz J, West KP, Khatry SK, LeClerk SC, Pradhan EK, Thapa MD, et al. Prevalence and risk factors for trachoma in Sarlahi district, Nepal. Br J Ophthalm 1996; 80:1037-41.

19. Scarpi MJ, Plutt RCA, Arruda HO. Prevalência de tracoma no povoado de Mocambo, Estado do Ceará, Brasil. Arq Bras Oftalmol 1989; 52:177-9.

20. Scarpi MJ, Silva RJM, Ferreira IA, Barbosa FAC, Plut RCA. Prevalência de tracoma em bairro do Município de Palmares, Estado de Pernambuco, Brasil. Arq Bras Oftalmol 1990; 53:171-4.

21. Scarpi MJ, Gentil R. Sinais e sintomas do tracoma em povoado do Estado da Bahia - Brasil. Arq Bras Oftalmol 1990; 53:276-8.

22. Moreira ATR, Scarpi MJ, Oyama C, Penteado R, Moreira LB, Guidugli T. Prevalência de tracoma no Município de Miraselva, Estado do Paraná, Brasil. Arq Bras Oftalmol 1993; 56:354-8.

23. Schémann JF, Sacko D, Malvy D, Momo G, Traore L, Bore O, et al. Risk fators for trachoma in Mali. Int J Epidemiol 2002; 31:194-201.

24. Detels R, Bailey RL, Dhir SP. Trachoma in Punjabi Indians in British Columbia: a prevalence study with comparisons to India. Am J Epidemiol 1966; 84:81-91.

25. Muñoz B, West S. Trachoma: the forgotten of blindness. Epidemiol Rev 1997; 19:205-17.

26. Mabey DCW, Solomon AW, Foster A. Trachoma. Lancet 2003; 363:223-9.

27. Güraksin A, Güllülü G. Prevalence of trachoma in Eastern Turkey. Int J Epidemiol 1997; 26:436-42.

28. Jha H, Chaudary JSP, Bhatta R, Miao Y, OsakiHolm S, Gaynor B, et al. Disappearance of trachoma from Western Nepal. Clin Infect Dis 2002; 35:765-8.

29. Schérmann JF, Guinot C, Ilboldo L, Momo G, Ko B, Sanfo O, et al. Trachoma, flies and environmental factors in Burkina Faso. Trans R Trop Med Hyg 2003; 97:63-8.

30. Sahlu T, Larson C. The prevalence and environmental risk factors for moderate and severe trachoma in southern Ethiopia. J Trop Med Hyg 1992; 95:36-41.

31. West KS. Blinding trachoma: prevention with the safe strategy. Am J Trop Med Hyg 2003; 69 Suppl 5:18-23.

32. Hsieh YH, Bobo LB, Quinn TO, West KS. Risk factors for trachoma: 6-year follow-up of children aged 1 and 2 years. Am J Epidemiol 2000; 152:20411 .

33. Thein J, Zhao P, Xu J, Jha H, Miao, Pizzarello L, et al. Does clinical diagnosis indicate ocular chlamydial infection in areas with a low prevalence of trachoma? Ophthalmic Epidemiol 2002; 9:263-9.

34. Szklo M, Javier Nieto F. Epidemiology: beyond the basics. Gaithersburg: Aspen Publishers; 2000.

35. Bailey R, Duong T, Carpenter R, Whittle H, Mabey D. The duration of human ocular Chlamydia trachomatis infection is age dependent. Epidemiol Infect 1999; 123:479-86.

Recebido em 25/Jun/2004

Versão final reapresentada em 27/Abr/2005

Aprovado em 16/Mai/2005 